\title{
Automated localisation of the optic disc, fovea, and retinal blood vessels from digital colour fundus images
}

\author{
Chanjira Sinthanayothin, James F Boyce, Helen L Cook, Thomas H Williamson
}

\begin{abstract}
Aim-To recognise automatically the main components of the fundus on digital colour images.

Methods-The main features of a fundus retinal image were defined as the optic disc, fovea, and blood vessels. Methods are described for their automatic recognition and location. 112 retinal images were preprocessed via adaptive, local, contrast enhancement. The optic discs were located by identifying the area with the highest variation in intensity of adjacent pixels. Blood vessels were identified by means of a multilayer perceptron neural net, for which the inputs were derived from a principal component analysis (PCA) of the image and edge detection of the first component of PCA. The foveas were identified using matching correlation together with characteristics typical of a fovea-for example, darkest area in the neighbourhood of the optic disc. The main components of the image were identified by an experienced ophthalmologist for comparison with computerised methods.
\end{abstract}

Results-The sensitivity and specificity of the recognition of each retinal main component was as follows: $99.1 \%$ and $99.1 \%$ for the optic disc; $83.3 \%$ and $91.0 \%$ for blood vessels; $80.4 \%$ and $99.1 \%$ for the fovea.

Conclusions-In this study the optic disc, blood vessels, and fovea were accurately detected. The identification of the normal components of the retinal image will aid the future detection of diseases in these regions. In diabetic retinopathy, for example, an image could be analysed for retinopathy with reference to sight threatening complications such as disc neovascularisation, vascular changes, or foveal exudation.

(Br F Ophthalmol 1999;83:902-910)

The patterns of disease that affect the fundus of the eye are varied and usually require identification by a trained human observer such as a clinical ophthalmologist. The employment of digital fundus imaging in ophthalmology provides us with digitised data that could be exploited for computerised detection of disease. Indeed, many investigators use computerised image analysis of the eye, under the direction of a human observer. ${ }^{1-4}$ The management of certain diseases would be greatly facilitated if a fully automated method was employed. ${ }^{5}$ An obvious example is the care of diabetic retinopathy, which requires the screening of large numbers of patients (approximately 30000 individuals per million total population ${ }^{6}$ ). Screening of diabetic retinopathy may reduce blindness in these patients by $50 \%$ and can provide considerable cost savings to public health systems. ${ }^{8}{ }^{9}$ Most methods, however, require identification of retinopathy by expensive, specifically trained personnel. ${ }^{10-13}$ A wholly automated approach involving fundus image analysis by computer could provide an immediate classification of retinopathy without the need for specialist opinions.

Manual semiquantitative methods of image processing have been employed to provide faster and more accurate observation of the degree of macula oedema in fluorescein images. ${ }^{14}$ Progress has been made towards the development of a fully automated system to detect microaneurysms in digitised fluorescein angiograms. ${ }^{15}{ }^{16}$ Fluorescein angiogram images are good for observing some pathologies such as microaneurysms which are indicators of diabetic retinopathy. It is not an ideal method for an automatic screening system since it requires an injection of fluorescein into the body. This disadvantage makes the use of colour fundus images, which do not require an injection of fluorescein, more suitable for automatic screening.

The detection of blood vessels using a method called 2D matched filters has been proposed. ${ }^{17}$ This method requires the convolution of each image with a filter of size $15 \times 15$ for at least 12 different kernels in order to search for directional components along distinct orientations. The large size of the convolution kernel entails heavy computational cost. An alternative method to recognise blood vessels was developed by Akita and Kuga. ${ }^{18}$ This work does not include automatic diagnosis of diseases, because it was performed from the viewpoint of digital image processing and artificial intelligence.

None of the techniques quoted above has been tested on large volumes of retinal images. They were found to fail for large numbers of retinal images, in contrast with the successful performance of a neural network.

Artificial neural networks (NNs) have been employed previously to examine scanned digital images of colour fundus slides. ${ }^{19}$ Using NNs, features of retinopathy such as haemorrhages and exudates were detected. These were used to identify whether retinopathy was present or absent in a screened population but 


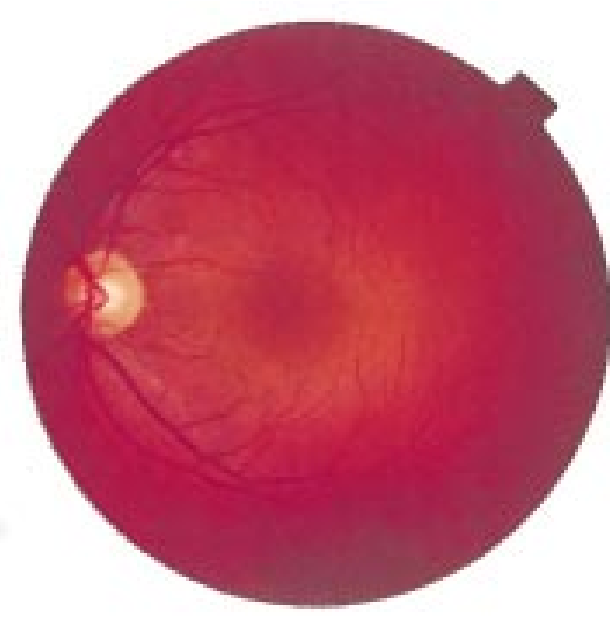

Figure 1 Digital colour retinal image.

allowed no provision for grading of retinopathy. The images with retinopathy still require a grading by a trained observer. Grading greatly improves the efficiency of the screening service, because only those patients with sight threatening complications are identified for ophthalmological management. This task is more complex than identifying the presence of retinopathy because the computer programme must be able to detect changes such as neovascularisation, cotton wool spots, vascular changes, and perifoveal exudation. ${ }^{20}$ The first step for achieving this aim is to be able to locate automatically the main regions of the fundusthat is, the optic disc, fovea, and blood vessels. The data from these regions can then be analysed for features of sight threatening disease. Identification of the regions of the fundus may also aid analysis of images for other diseases that affect these areas preferentiallyfor example, glaucoma and senile macular degeneration.

In this study, a variety of computer image analysis methods, including NNs, were used to analyse images to detect the main regions of the fundus.

\section{Methods}

In all, 112 TIF (tagged image format) images of the fundi of patients attending a diabetic screening service were obtained using a Topcon TRC-NW5S non-mydriatic retinal camera. Forty degree images were used.

\section{PREPROCESSING OF COLOUR RETINAL IMAGES}

The captured fundus images were of dimensions $570 \times 550$ pixels. Each pixel contained three values, red, green, and blue, each value being quantised to 256 grey levels. An example can be seen in Figure 1. The contrast of the fundus images tended to diminish as the distance of a pixel from the centre of the image increased. The objective of preprocessing was to reduce this effect and to normalise the mean intensity. The intensities of the three colour bands were transformed to an intensity-huesaturation representation. ${ }^{21}$ This allowed the intensity to be processed without affecting the perceived relative colour values of the pixels.

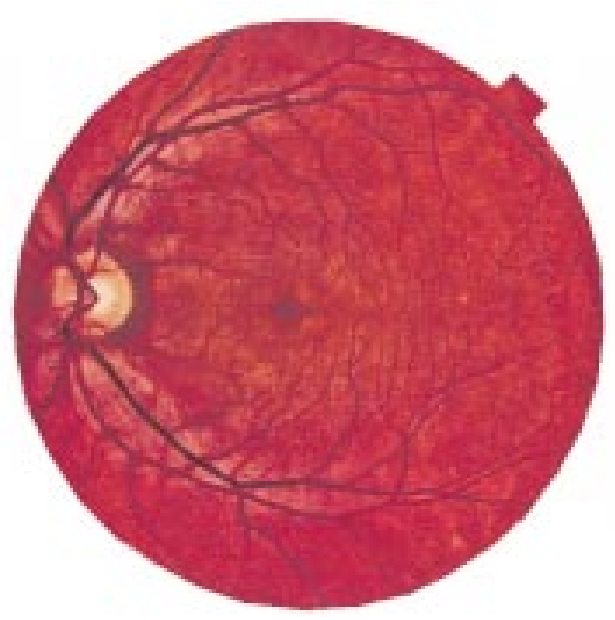

Figure 2 Retinal image after preprocessing by local colour contrast enhancement.

The contrast of the intensity was enhanced by a locally adaptive transformation. Consider a subimage, $W(i, j)$, of size $M \times M$ pixels centred on a pixel located at $(i, j)$. Denote the mean and standard deviation of the intensity within $W(i, j)$ by $\langle f\rangle_{W}$ and $\sigma_{W}$ respectively. Suppose that $f_{\max }$ and $f_{\min }$ were the maximum and minimum intensities of the whole image.

The adaptive local contrast enhancement transformation was defined by equations (A1) and (A2) of appendix A.

Figure 2 shows the effect of preprocessing on a fundus image, Figure 1.

RECOGNITION OF THE OPTIC DISC

The optic disc appeared in the fundus image as a yellowish region. It typically occupied approximately one seventh of the entire image, 80 $\times 80$ pixels. The appearance was characterised by a relatively rapid variation in intensity because the "dark" blood vessels were beside the "bright" nerve fibres. The variance of intensity of adjacent pixels was used for recognition of the optic disc.

Consider a subimage $W(i, j)$ of dimensions $M \times M$ centred on pixel $(i, j)$. Let $\langle f\rangle w(i, j)$ as defined by equation $(A 3)$ be the mean intensity within $W(i, j)$. (If $W(i, j)$ extended beyond the image, then undefined intensities were set to zero and the normalisation factor was correspondingly reduced.)

A variance image was formed by the transformation

$g(i, j) \rightarrow p(i, j)=\left\langle f^{2}\right\rangle_{W}-\left(\langle f\rangle_{W}\right)^{2}$

where the subimage was $80 \times 80$ pixels. An image of the average variance within subimages was then obtained as

$p(i, j) \rightarrow q(i, j)=\langle p\rangle_{W(i, j)}$.

The location of the maximum of this image was taken as the centre of the optic disc, $\left(i_{d}, j_{d}\right)$,

$$
\left(i_{d}, j_{d}\right)=\arg \max _{W(i, j)}<p>_{W(i, j)}
$$

The variance image of Figure 2 is shown in Figure 3 and the location of the optic disc in Figure 7. 


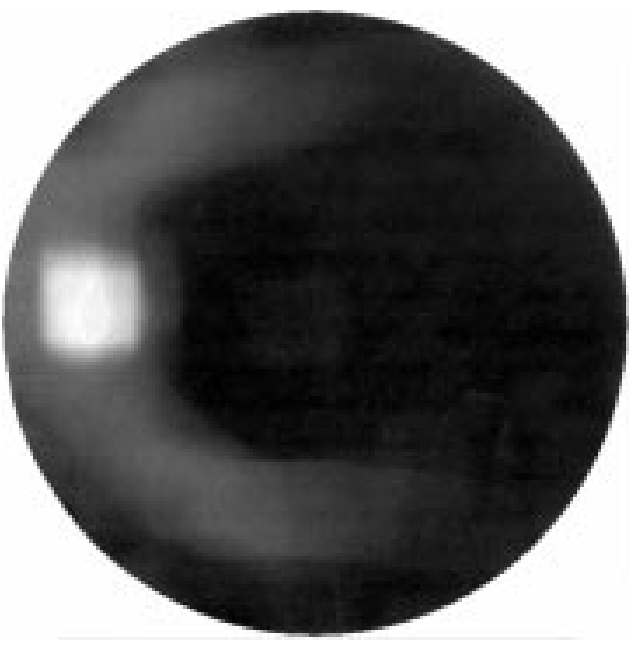

Figure 3 The variance image of Figure 2.

RECOGNITION OF BLOOD VESSELS

Blood vessels appeared as networks of either deep red or orange-red filaments that originated within the optic disc and were of progressively diminishing width. A multilayer perceptron NN was used to classify each pixel of the image. ${ }^{22-24}$ Preprocessing of the image was necessary before presentation to the input layer of the NN. Pattern classifiers are most effective when acting on linearly separable data in a small number of dimensions. More details are described in appendix B.

The values of the three spectral bands of a pixel were strongly correlated. The principal component transformation ${ }^{25}$ was used to rotate the axes from red-green-blue to three orthogonal axes along the three principal axes of correlation, thus diagonalising the correlation coefficient matrix. The values along the first axis exhibited the maximum correlated variation of the data, containing the main structural features. Uncorrelated noise was concentrated mainly along the third axis while, in general, texture tended to be along the second. The original data were reduced in dimensionality by two thirds by the principal component transformation.

A measure of edge strength was obtained for each pixel by processing the image from the first principal component using a Canny edge operator. ${ }^{26-28}$ This was used to enhance vessel/ non-vessel separability.
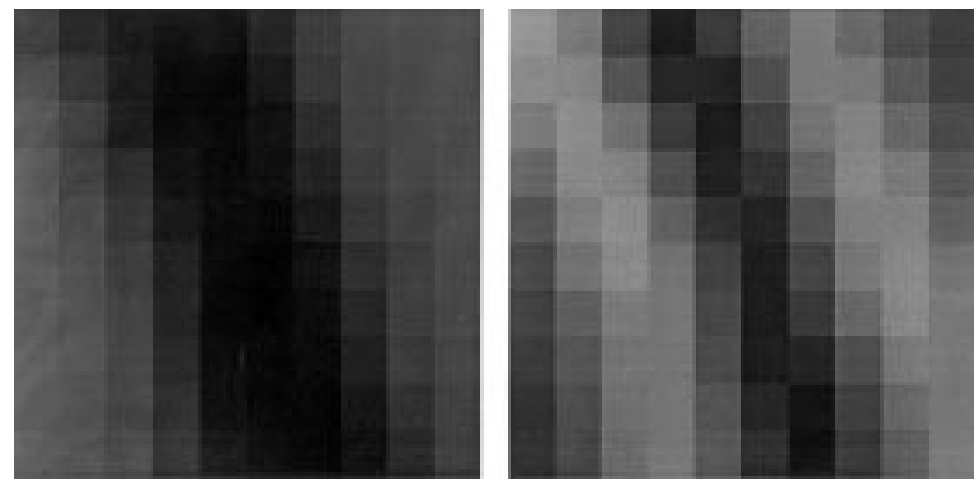

Figure 4 An example of the data input to the net, of size $2 \times 10 \times 10$ pixels. In this example, the pattern was classified as vessel.

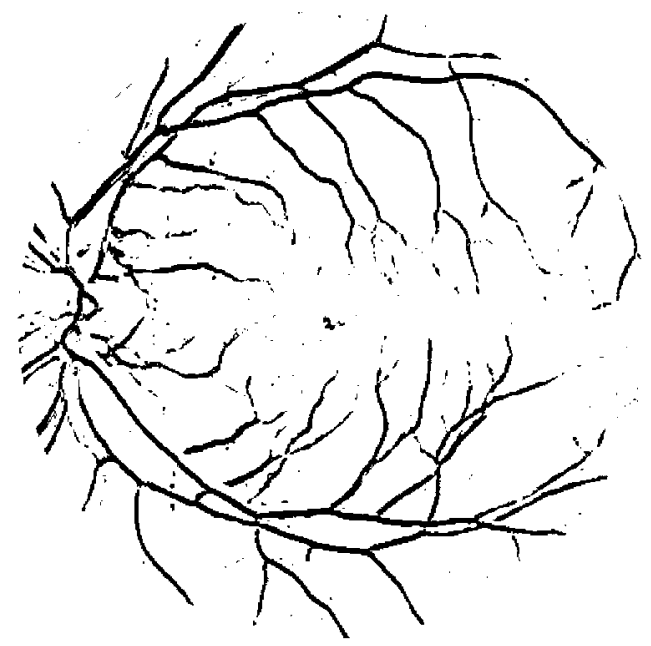

Figure 5 Classification of the image Figure 2 into vessels/non-vessels.

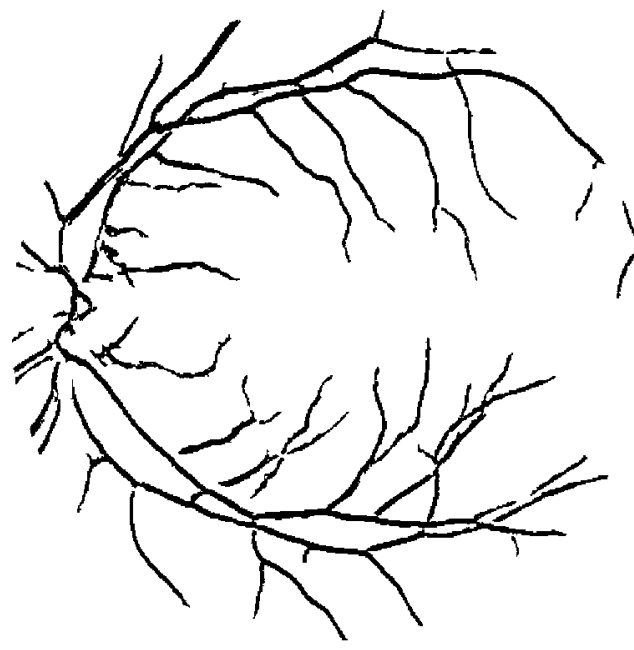

Figure 6 The classified image after post-processing to remove small regions.

NEURAL NETWORK ALGORITHM

Each pixel of a fundus image was classified as vessel or non-vessel. The data input to the $\mathrm{NN}$ were the first principal componen $\mathrm{t}^{29}$ and edge strength values from a subimage of $10 \times 10$ pixels localised on the pixel being classified, as shown in Figure 4. The net was a three layer perceptron having 200 input nodes, 20 hidden nodes, and two output nodes.

A training/validation data set of 25094 examples, comprising 8718 vessel and 16376 non-vessel, was formed by hand and checked by a clinician. The back propagation algorithm with early stopping was applied, ${ }^{30} 31$ using $5 / 6$ of the data for training and $1 / 6$ for validation.

POST-PROCESSING ${ }^{32} 33$

Figure 5 shows the classification of the entire image into vessel and non-vessel, denoted as black and white respectively.

The performance of the classifier was enhanced by the inclusion of contextual (semantic) conditions. Small isolated regions of pixels that were misclassified as blood vessels were reclassified using the properties that vessels occur within filaments, which form networks. Three criteria were applied-size, com- 


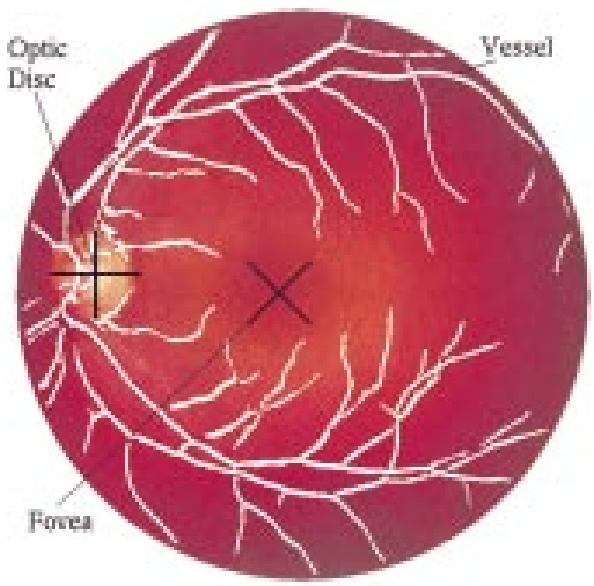

Figure 7 The results of automatic recognition of the main components of the fundus from a digital fundus colour image.

pactness, and shape. Regions smaller than 30 pixels were reclassified as non-vessels. The compactness of a region may be expressed using the ratio of the square of the perimeter to the area ${ }^{34}$-for example, circular discs have a ratio of $4 \pi$. Regions whose ratios were less than 40 were reclassified as non-vessels. Approximating a region by an elliptical disc yields a
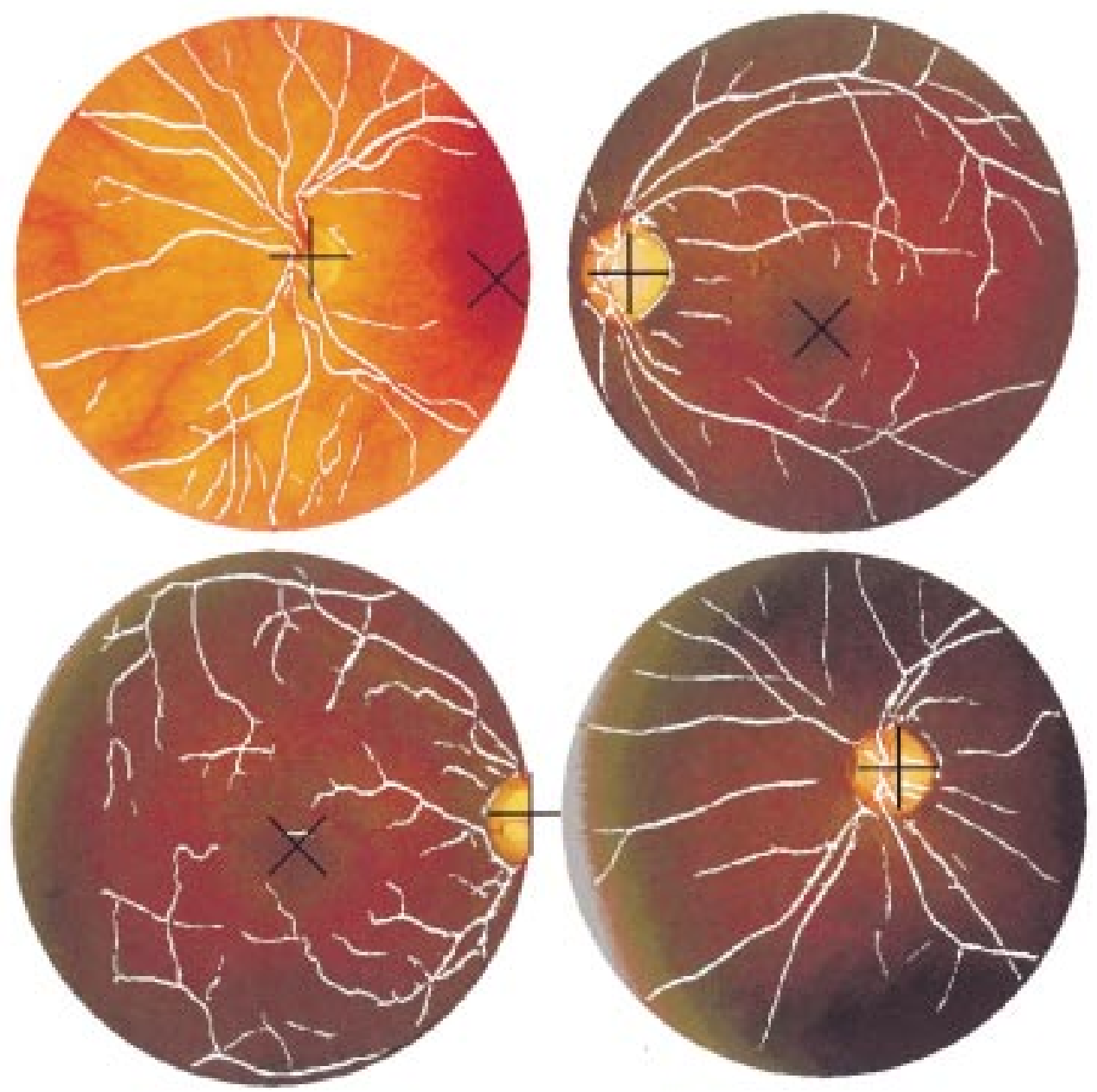

measure of shape in terms of the ratio of the major and minor axes. ${ }^{35}$ Regions smaller than 100 pixels with a ratio smaller than 0.95 were reclassified as non-vessels as can be seen in appendix C. Figure 6 shows the effect of such post-processing on an image.

RECOGNITION OF THE FOVEA

The centre of the fovea was usually located at a distance of approximately 2.5 times the diameter of the optic disc, from the centre of the optic disc. It was the darkest area of the fundus image, with approximately the same intensity as the blood vessels. The fovea was first correlated to a template of intensities. The template was chosen to approximate a typical fovea and was defined by

$$
g(i, j)=128\left[1-\frac{1}{2} \exp \left(\frac{-\left(i^{2}+j^{2}\right)}{2 \sigma^{2}}\right)\right]
$$

where $(i, j)$ are relative to the centre of the template. A template of size $40 \times 40$ pixels was employed, the standard deviation of the Gaussian distribution being $\sigma=22$. Given a subimage $W(i, j)$ centred on pixel $(i, j)$ of dimensions $M \times M$ with intensities $g(k, l),(k, l) \in W(i, j)$, the

Figure 8 A sample of images showing the results of the recognition of the main components from digital fundus colour images. 
correlation coefficient of $W$ at $(i, j)$ with an image having intensities $f(i, j)$ is ${ }^{21}$

$\gamma(i, j)=\frac{\sum_{k, l}\left[f(k, l)-\langle f\rangle_{W}\right]\left[g(i-k, j-l)-<g>_{W}\right]}{\left\{\sum_{k, l}\left(f(k, l)-\langle f\rangle_{W}\right)^{2} \sum_{k, l}\left(g(i-k, j-l)-<g>_{W}\right)^{2}\right\}^{1 / 2}}$

The correlation coefficient $\gamma(i, j)$ is scaled to the range $[-1 \leqslant \gamma \leqslant 1]$, and is independent of mean or contrast changes in $f(i, j)$ and $g(i, j)$. The range of values runs from anti-correlation, -1 , through no correlation, 0 , to perfect correlation +1 .

The location of maximum correlation between the template and the intensity image, obtained from the intensity-hue-saturation transformation, was chosen as the location of the fovea, subject to the condition that it be an acceptable distance from the optic disc and in a region of darkest intensity.

The criteria deciding the existence of the fovea were a correlation coefficient more than 0.5 and a location at the darkest area in the allowed neighbourhood of the optic disc.

Figures 7 and 8 give examples of foveal location, the cross indicates the located position in each example.
VALIDATION OF THE ACCURACY OF DETECTION OF REGIONS

An experienced ophthalmologist observed the localisation of the optic disc and fovea by the algorithms. In order to provide independent test data for the blood vessels, the same ophthalmologist manually traced the vessels of 73 randomly selected patches of $20 \times 20$ pixels taken from the images. The traced vessels were then compared with the positions of the vessels identified by the neural network. An example of a random patch is shown in Figure 9.

\section{Results}

The recognition rates for optic disc, blood vessels and fovea were as follows:

(1) The optic disc was identified incorrectly in one image. The sensitivity and specificity for optic disc recognition were $99.1 \%$ and $99.1 \%$ respectively.

(2) The recognition rate of blood vessels by the neural network was $99.56 \%$ for training and $96.88 \%$ for validation data respectively. The sensitivity and specificity of the detection of blood vessels were calculated for each of the 73 patches. The overall sensitivity and specificity for the detection of

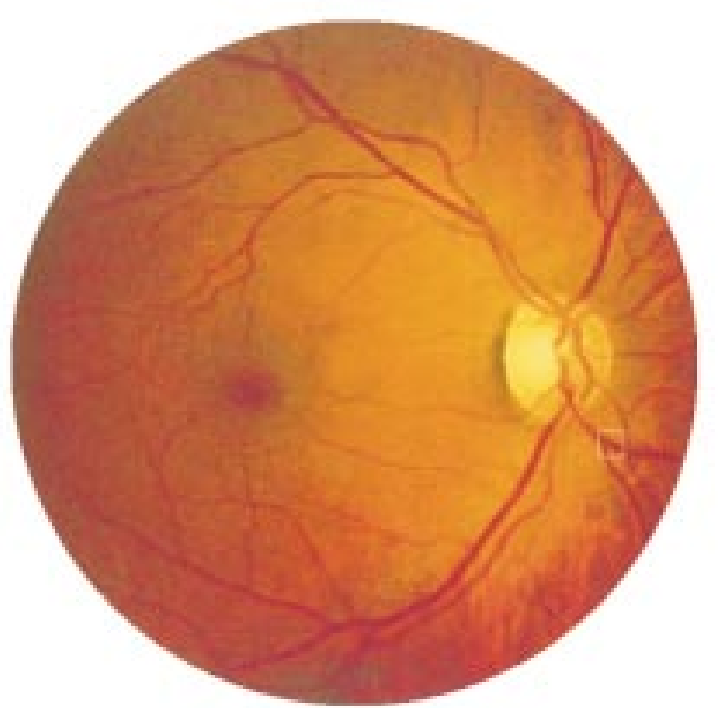

Example of $20 \times 20$ sampling patch image. (In the white square box)

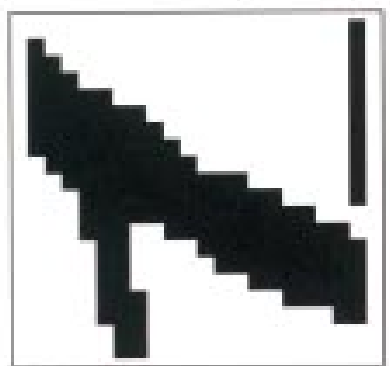

Vessels labeled by clinician.

Vessels by clinician \& NN

Blue: Non-vessels by clinician but vessels by $\mathrm{NN}$.

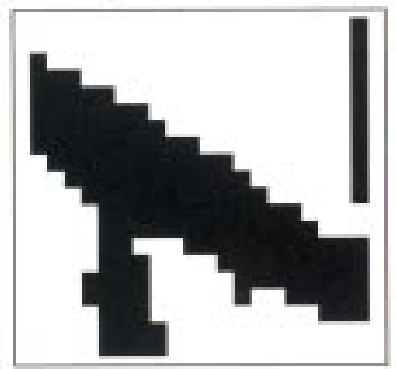

Yexels labeled by Neural Network. (NN).

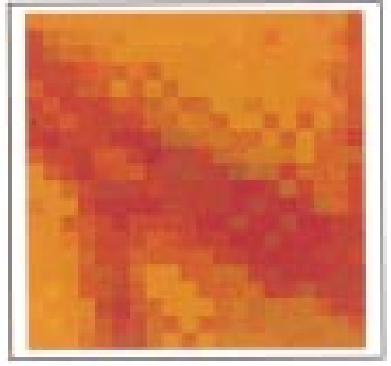

Expand patch imaze.

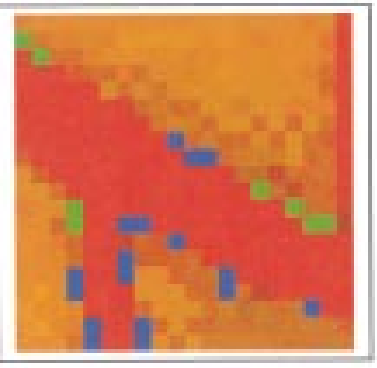

Comparisoce of labels from clinician and $\mathrm{NN}$.

Gireen: Vessels by elinician but non-vessels by $\mathrm{NN}$.

Figure 9 Example of patch of size $20 \times 20$ pixels used to measure the accuracy of vessels recognition. 
the blood vessels were $83.3 \%$ (SD 16.8\%) and $91.0 \%(5.2 \%)$ respectively.

(3) To assess the accuracy of recognition of the fovea, the images were separated into three groups: group 1: 71 images presented all of the fovea of within the image. In 60 the fovea was detected correctly $(84.5 \%)$ but in $11(15.5 \%)$ the fovea was unidentified; group 2: in 29 retinal images the fovea was at the edge of the image but more than half was present on the image. In 18 images the correct position of the fovea was located $(62.1 \%)$. In one image the fovea was detected inaccurately in the nasal retina $(3.4 \%)$. The fovea was not identified in 10 images (34.5\%); group 3 : 12 retinal images either presented no fovea or less than half of the foveal area was within the image. The algorithm did not identify a fovea in these images.

The overall sensitivity and specificity of the detection of the fovea were $80.4 \%$ and $99.1 \%$ respectively.

\section{Discussion}

In this study computer based algorithms were used to detect the main regions of the fundus without any intervention from an operator. The accuracy of the detection was high for the optic disc, blood vessels, and the fovea (especially when the image contained the whole foveal area). It is hoped that the detection of these regions will aid the examination of fundal disorders. The optic disc was particularly reliably detected and may be examined in the future for patterns of disease such as glaucoma. ${ }^{36}$ The fovea was missed in a number of cases but usually when there was poor centration of the fovea in the image. This can be easily remedied by more careful fundus photography. The detection of the major blood vessels was performed using $\mathrm{NN}$ analysis. NNs have been employed in the past in other areas of medicine ${ }^{37}$ and ophthalmology ${ }^{38} 39$ because of the capability of these programs to cope with highly variable images. Indeed, NN have already been used before to detect features of retinopathy but employing minimal preprocessing. ${ }^{40}$ The preprocessing and postprocessing used in this study reduces the reliance upon the $\mathrm{NN}$ and improves the efficiency of the computer analysis. The smaller blood vessels were more difficult to detect. However, the method used to calculate the accuracy of the detection of the blood vessels by comparing vessels recognised by the $\mathrm{NN}$ technique with the vessels traced by the ophthalmologist may introduce some errors. It was technically difficult for the ophthalmologist to locate subjectively the exact position of vessels, especially at their edges. Therefore, the accuracy of the ophthalmologists' identification of blood vessels may have been variable. Other methods may need to be explored in order to detect all of the blood vessels-for example, active contour models (SNAKES), ${ }^{41}{ }^{42}$ to avoid confusion with features such as retinal haemorrhages. There may be other applications for this technology-for example, the accurate detection of the major blood vessels may allow the "fingerprint" of the blood vessels to be used for identifying individuals in a way similar to that in which iris crypts have been utilised. ${ }^{43}$

In this study it was possible to detect the main regions of the fundus image. Once these have been identified, the data from these regions can be analysed for abnormality. Of course some of the diseases that we would propose to study may alter the appearance of these regions, reducing their detection. However, the algorithms were designed to minimise this risk, particularly for disease processes such as diabetic retinopathy. In diabetic retinopathy further algorithms will be required to detect features which indicate risk to the patient's sight such as neovascularisation, cotton wool spots, venous changes, and parafoveal exudation. The detection of other features of retinopathy in diabetes (haemorrhages and exudates), and other disorders such as senile macular degeneration, will be facilitated by the removal from the image data set of complex regional features such as the blood vessels and the optic disc. In diabetes, grading of a patient's retinopathy by fundus imaging and computer analysis, at the site of acquisition of the image, would allow an immediate opinion for the patient on the urgency of referral for an ophthalmological opinion.

In conclusion, computer algorithms were able to detect regions of the fundus. These may be exploited for the examination of patterns of disease. This may have particular relevance to the management of common ophthalmological disorders such as diabetic retinopathy.

\section{Appendix A: colour local contrast enhancement}

The $R G B$ colours, where $R, G$, and $B$ are abbreviated from the colours Red, Green, and Blue respectively, represent the colour model used for computer graphics or image analysis. Another colour model, which will be used in this work, is the IHS model, where $I, H$, and $S$ are abbreviations for Intensity, Hue, and Saturation respectively. The $R G B$ and $I H S$ have an invertible relation between them. ${ }^{21}$

The importance of an $R G B$ model is to display colour images. To present the full colour techniques for image enhancement in some detail, we are interested in the IHS model. The IHS model is best suited to present full colour techniques for image enhancement in detail. It is important that once the IHS model is applied to enhance an image, it must be converted back to $R G B$ for visual display. The IHS model is suitable for image enhancement because the intensity component is decoupled from the colour information of the image. Applying the local contrast enhancement technique to the intensity component and converting the result to $R G B$ for display will not affect the colour content of the image.

LOCAL CONTRAST ENHANCEMENT ${ }^{44}$

Let the intensity, $f$, of the picture elements (pixels) of an $N \times N$ digital image be indexed by $(i, j) 1 \leqslant i, j \leqslant N$. Consider a subimage of size 
$M \times M$ centred on $(i, j)$ in this paper $M=49$. Denote the mean and standard deviation of the intensity within $W$ by $\langle f\rangle_{\mathrm{w}}$ and $\sigma_{\mathrm{w}}(f)$ respectively.

The objective is to define a point transformation dependent on $W$ such that the distribution is localised around the mean of the intensity and covers the entire intensity range. The implicit assumption is that $W$ is large enough to contain a statistically representative distribution of the local variation of grey levels, yet small enough to be unaffected by the gradual change of contrast between the centre and the periphery of the fundus image. The adaptive contrast enhancement transformation is defined by

$$
f(i, j) \rightarrow g(i, j)=225 \frac{\left[\Psi_{W}(f)-\Psi_{W}\left(f_{\min }\right)\right]}{\left[\Psi_{W}\left(f_{\max }\right)-\Psi_{W}\left(f_{\min }\right)\right]}
$$

where the sigmoidal function was

$$
\Psi_{W}(f)=\left[1+\exp \left(\frac{\langle f\rangle_{W}-f}{\sigma_{W}}\right)\right]^{-1}
$$

while $f_{\max }$ and $f_{\min }$ are the maximum and minimum values of intensity within the whole image with

$$
\begin{aligned}
f>_{W(i, j)} & =\frac{1}{M^{2}} \sum_{(k, l) \in W(i, j)} f(k, l) \\
\sigma_{W}^{2}(f) & =\frac{1}{M^{2}} \sum_{(k, l) \in W(i, j)}\left(f(k, l)-\langle f\rangle_{W}\right)^{2}
\end{aligned}
$$

The local contrast enhancement function provides large contrast enhancement for an initially small $\sigma$ (poor contrast) and little contrast enhancement for an initially large $\sigma$ (high contrast).

As a result of local contrast enhancement, the dark area is brighter and clearer showing more detail. However, the technique of local contrast enhancement not only adjusts the contrast of the image but also increases the noise. Hence, a 2D Gaussian smoothing filter or median filter has been applied in order to reduce the noise before the local contrast enhancement process.

\section{Appendix B: prepare data for NN \\ FIRST PRINCIPAL COMPONENT ${ }^{45}$}

The PCA (principal component analysis) technique is the same as the Karhunen Loeve transform technique, also known as Hotelling transform, which aims to form linear combinations of the image bands in order to maximise the information content in the highest order bands. Giving a set of $K$ image bands, denoted by the intensities of $\mathrm{N}^{2}$ pixels $f_{\mathrm{a}}(i)$ for $i=1, \ldots, N a=$ $1, \ldots, K$. We first form a set of zero mean images by subtracting off the mean of each band

$$
g_{a}=f_{a}(i)-<f_{a}(i)>
$$

where

$$
<f_{a}(i)>=\frac{1}{N^{2}} \sum_{i=1}^{N^{2}} f_{a}(i)
$$

To simplify the formulation, it is convenient to write the set of $K$ image bands $g_{a}(i)$ as a $N^{2} \times$ $K$ matrix, where each image of the spectral band forms a column of the matrix normally,

$$
G=\left[\begin{array}{cccc}
g_{1}(1) & g_{2}(1) & \cdots & g_{K}(1) \\
g_{1}(2) & g_{2}(1) & \cdots & g_{K}(2) \\
\vdots & \vdots & \ddots & \vdots \\
g_{1}\left(N^{2}\right) & g_{2}\left(N^{2}\right) & \cdots & g_{K}\left(N^{2}\right)
\end{array}\right]
$$

We define a $K$ by $K$ matrix $C$ as $C=G^{T} G$,

where $G^{\mathrm{T}}$ is the transpose of $G$. The matrix $C$, can be expressed in terms of the input image $g_{\mathrm{a}}(i)$ as having elements,

$$
\begin{aligned}
c_{a b} & =\sum_{i=1}^{N^{2}} g_{a}(i) g_{b}(i) \\
& =\sum_{i=1}^{N^{2}}\left(f_{a}(i)-<f_{a}(i)>\right)\left(f_{b}(i)-<f_{b}(i)>\right) \\
& =\sum_{i=1}^{N^{2}} f_{a}(i) f_{b}(i)-\frac{1}{N^{2}} \sum_{i=1}^{N^{2}} f_{a}(i) \sum_{i=1}^{N^{2}} f_{b}(i)
\end{aligned}
$$

This is the un-normalised correlation between the $a$ th and the $b$ th image bands.

Hence $C$ is the spectral correlation matrix of the images. We form a matrix $H$ of uncorrelated images by the orthogonal $K \times K$ matrix transform $B$.

$H=G B$

Since the columns of $H$ are uncorrelated

$H^{T} H=\Lambda$

where $\Lambda$ is a diagonal matrix

$$
\Lambda=\left[\begin{array}{cccc}
\lambda_{1} & 0 & \cdots & 0 \\
0 & \lambda_{2} & \cdots & 0 \\
\vdots & \vdots & \ddots & \vdots \\
0 & 0 & \cdots & \lambda_{K}
\end{array}\right]
$$

it follows that

$C B=B \Lambda$

The above equation is just the familiar eigen vector/value problem where the $\lambda_{\mathrm{a}}$ are the eigenvalues of the matrix $C$ and the columns of the matrix $B$ are the corresponding eigenvectors. Since the matrix $C$ is symmetric, $c_{\mathrm{ab}}=c_{\mathrm{ba}}$, the eigenvector problem involves finding the eigenvalues and vectors of a real symmetric matrix. Hence we can solve for the eigenvalues and vectors by applying Jacobi transformations. ${ }^{46}$ Finally, the transform of the set of $K$ orthogonal images $h_{\mathrm{a}}(i)$, being linear combinations of the normalised images $g_{\mathrm{a}}(i)$, is given by

$$
h_{a}(i)=\sum_{b=1}^{K} b_{a b} g_{a}(i)
$$

for $a=1, \ldots, K$ and $h_{1}(1), h_{1}(2), \ldots, h_{1}(N)$ will be the first component of the set of principal components which will be used together with the edge gradient as the pattern data for classification by a neural network.

EDGE GRADIENT

In this paper we applied the edge operator to the first component of a PCA image. Canny has defined an edge detection operator, which is optimal for step edges corrupted by white noise. The operator that we use in this work is 
the implementation of operator obtained by Spacek. The reasons that we use Spacek's solution because Canny was unable to construct a solution of the defining conditions and so approximated one by a difference of gaussians. Spacek demonstrated that performance of his solution was superior to Canny's approximation. Since the operator satisfies the conditions proposed by Canny we refer to it as the Canny edge operator.

The Spacek method that we use takes the form. ${ }^{28}$

$h(x)=16.9564 \sin (x)+13.0161 \cos (x)-$ $18.8629 \exp (x)+4.8468 \exp (-x)+1 \quad$ (B11) $h(x)$ has been defined in the interval $(-1,0)$. Values in the range $(0,1)$ may be obtained from the anti-symmetry of the edge operator. For our application, we have modified to use the Canny edge filter in two dimensions by smoothing the operator in the orthogonal direction, hence yielding two dimensional edge kernels $h_{\mathrm{x}}(x, y)$ and $h_{\mathrm{y}}(x, y)$ for edge strengths in the $x, y$ directions respectively. Now we apply the $2 \mathrm{D}$ edge filter in $x$ and $y$ directions. The response of the filters to the image is given by a $2 \mathrm{D}$ convolution integral.

The response an image to an edge kernel is given by a $2 \mathrm{D}$ discrete convolution, the edge strength in the $x$ direction

$e_{x}(x, y)=\sum \sum h_{x}(\xi, \eta) f(x-\xi, y-\eta) d \xi d \eta \quad$ (B12) with a similar equation for $e_{y}(x, y)$. The intensity of the edge image intensity is defined by

$$
e(x, y)=\left[\left(e_{x}(x, y)\right)^{2}+\left(e_{y}(x, y)\right)^{2}\right]^{1 / 2}
$$

\section{Appendix C: post-processing}

AREA

The simplest and most natural property of a region is its area, given by the number of pixels of which the region is comprised. This method was used to remove the small regions of the output vessels/non-vessels classification from neural net, small regions with less than 30 pixels were removed.

COMPACTNESS $^{33}$

Compactness is a commonly used shape descriptor independent of linear transformations given by

$$
\text { compactness }=\frac{(\text { region border length })^{2}}{\text { area }}
$$

The most compact region in Euclidean space is a circle. Compactness assumes values in the interval $(1, \infty)$ in digital images if the boundary is defined as an inner boundary. The limit value of compactness in this work is 40 , within the region less than 100 pixels will be determined.

\section{ELLIPSE PARAMETER}

The ellipse parameter used is the ratio between the major and the sum of the two (major and minor) axes of an ellipse. This parameter describes the shape of the region and lies between 0.5 (a circle) and 1 (a line). The method that we used to calculate this parameter is the same as employed in PCA. Considering a region, with two variables, $x$ variable (positions of the region pixels in $x$ coordinate) and $y$ variable (positions of the region pixels in $y$ coordinate). The major and minor axes of the region can be calculated as the eigen values of the covariance matrix:

$$
C=\left[\begin{array}{cc}
\sum\left(x_{i}-<x>\right)^{2} & \sum\left(x_{i}-<x>\right)\left(y_{i}-<y>\right) \\
\sum\left(x_{i}-<x>\right)\left(y_{i}-<y>\right) & \sum\left(y_{i}-<y>\right)^{2}
\end{array}\right]
$$

where $x_{\mathrm{i}}, y_{\mathrm{i}}$ are coordinates in the $x, y$ directions of the region and $\langle x\rangle,\langle y\rangle$ are the average value of the region's $x, y$ coordinates. From this property, the ellipse parameter of circle, ellipse, and line are $0.5,0.5<$ ellipse param $<1$, and 1 respectively. From our experiments with the test images of variable shapes, we decided to use an ellipse parameter of less than 0.95 the small regions (less than 100 pixels) to classify then as non-vessels.

We acknowledge the help of Professor Sonksen, Robin Edwards, and Shirley Smith of the Department of Medicine, St Thomas's Hospital, London, for the use of their images.

Grant support organisation: The Development and Promotion of Science and Technology Talent's Project (Thailand); Overseas Research Student Awards (UK)

Proprietary interest category: Nil.

1 Katz N, Goldbaum M, Nelson M, et al. An image processing system for automatic retina diagnosis. SPIE 1988;902:131-

2 Spencer T, Phillips RP, Sharp PF, et al. Automated detection and quantification of microaneurysms in fluorescein angiograms. Graefes Arch Clin Exp Ophthalmol 1992;230: $36-41$.

3 Ward NP, Tomlinson S, Taylor CJ. Image analysis of fundus photographs. The detection and measurement of exudates associated with diabetic retinopathy. Ophthalmology 1989; 96:80-6.

4 Phillips RP, Spencer T, Ross PG, et al. Quantification of diabetic maculopathy by digital imaging of the fundus. Eye 1991;5:130-7.

5 Williamson TH, Keating D. Telemedicine and computers in diabetic retinopathy screening (commentary). $\mathrm{Br} f \mathrm{Oph}$ thalmol 1998;82:5-7.

6 Singer DE, Nathan DM, Fogel HA, et al. Screening for diabetic retinopathy. Ann Intern Dis 1992;116:660-71.

7 Retinopathy Working Party. A protocol for screening for diabetic retinopathy in Europe. Diabetic Med 1991;8:263-7.

8 Wareham NJ. Cost-effectiveness of alternative methods for diabetic retinopathy screening (letter). Diabetes Care 1993; 16:844.

9 Javitt JC, Canner JK, Sommer A. Cost effectiveness of current approaches to the control of retinopathy in type I diabetics. Ophthalmology 1989;96:255-64.

10 Williams R, Nussey S, Humphrey R, et al. Assessment of non-mydriatic photography in detection of diabetic retinopathy. BMF 1986;293:1140-2

11 Higgs ER, Harney BA, Kelleher A, et al. Detection of diabetic retinopathy in the community using a nonmydriatic camera. Diabetic Med 1991;8:551-5.

12 Taylor R, Lovelock L, Tunbridge WM, et al. Comparison of non-mydriatic retinal photography with ophthalmoscopy in patients: mobile retinal camera study. BMf 1990;301: 1243-7.

13 Pugh JA, Jacobson JM, Van Heuven WA, et al. Screening for diabetic retinopathy. The wide-angle retinal camera. Diabetes Care 1993;16:889-95.

14 Phillips RP, Spencer T, Ross PGB, et al. Quantification of diabetic maculopathy by digital imaging of the fundus. Eye 1991;5:130-7.

15 Cree MJ, Olson JA, McHardy KC, et al. A fully automated comparative microaneurysm digital detection system. Eye 1997;11:622-8.

16 Spencer T, Phillips RP, Sharp PF, et al. Automated detection and quantification of microaneurysms in fluorescein angiograms. Graefes Arch Clin Exp Ophthalmol 1992;230: $36-41$

17 Chaudhuri S, Chatterjee S, Katz N, et al. Detection of blood vessels in retinal images using two-dimensional matched filters. IEEE Trans Med Imag 1989;8:263-9.

18 Akita K, Kuga H. A computer method of understanding ocular fundus images. Pattern Recognition 1982;15:431-43.

19 Gardner GG, Keating D, Williamson TH, et al. Detection of diabetic retinopathy using neural network analysis of fundal images. Neural Networks and Expert Systems in Medicine and Health Care (University of Plymouth) 1996:28-35.

20 Lee VS, Kingsley RM, Lee ET, et al. The diagnosis of diabetic retinopathy. Ophthalmoscopy versus fundus photography. Ophthalmology 1993;100:1504-12.

21 Gonzalez RC, Woods RE. Digital image processing. Reading: Addison-Wesley, 1993:229-37, 583-6.

22 Toulson DL, Boyce JF. Image segmentation using a neural network. IEE Colloquium on 3-D Imaging Techniques For Medicine, April 1991. 
23 Toulson DL, Boyce JF. Segmentation of MR images using neural nets. Proc Br Machine Vision Conf 1991:284-92.

24 Toulson DL, Boyce JF. Segmentation of MR images using neural nets (expanded version). Image Vis Comput 1992;10 $324-8$

25 Daultrey S. Principal components analysis. Norwich: Geo Abstracts Ltd, University of East Anglia 1976:3-26.

26 Canny J. A computational approach to edge detection. IEEE Trans Pattern Analysis and Machine Intelligence 1986;PAMI8-6:679-98.

27 Marr D, Hildreth E. Theory of edge detection. Proc Roy Soc Lond 1980;B207:187-217.

28 Spacek LA. The detection of contours and their visual motion. PhD Thesis, University of Essex 1985:59-66.

29 Oja E. Neural networks, principal components and subspaces. Int $\mathcal{F}$ Neural Syst 1989;1:61-8.

30 Lo SC, Freedman MT, Lin JS, et al. Automatic lung nodule detection using profile matching and back-propagation detection using profile matching and back-propagation

31 Astion ML, Wilding P. The application of backpropagation neural networks to problems in pathology and laboratory neural networks to problems in pathology and labor

32 Haddon JF, Boyce JF. Image segmentation by unifying region and boundary information. IEEE Trans Pattern region and boundary information. IEEE Trans Patte

33 Brelstaff GJ, Ibson MC, Elliott PJ. Edge-region integration for segmentation of MR images. Proc Br Machine Vision Conference (BMVC-90) 1990:139-44.

34 Sonka M, Hlavae V, Boyle R. Image processing, analysis and machine vision. Cambridge: Chapman \& Hall Computing, 1995:222-4, 227-8.

35 Goddard J, Kirby A. An introduction to factor analysis. Concepts and techniques in modern geography. Norwich: Institute of British Geographers, 1976:18-23.
36 Goldbaum $\mathrm{MH}$, Sample PA, White $\mathrm{H}$, et al. Interpretation of automated perimetry for glaucoma by neural network. Invest Ophthalmol Vis Sci 1994;35:3362-73.

37 Wu Y, Giger ML, Doi K, et al. Artificial neural networks in mammography: application to decision making in the diagnosis of breast cancer. Radiology 1993;187:81-7.

38 Spenceley SE, Henson DB, Bull DR. Visual field analysis using artificial neural networks. Ophthal Physiol Opt 1994;14:239-48.

39 Keating D, Mutlukan E, Evans A, et al. A back propagation neural network for the classification of visual field data. Phys Med Biol 1993;38:1263-70.

40 Gardner GG, Keating D, Williamson TH, et al. Automatic detection of diabetic retinopathy using an artificial neural network: a screening tool. Br F Ophthalmol 1996;80:940-4 (comment in Br f Ophthalmol 1996;80:937-8).

41 Kass M, Witkin A, Terzopoulos D. Snakes: active contour models. Int 7 Comp Vis 1988;1:321-31.

42 McInerney T, Terzopoulos D. Topologically adaptable snakes. Proc 5th Conf Computer Vision 1995:840-5.

43 Daugman JG. High confidence visual recognition of persons by a test of statistical independence. IEEE Trans Pattern Analysis Machine Intelligence 1993;15:1148-61.

44 Newsom RSB, Sinthanayothin C, Boyce J. Contrast enhancement for oral fluorescein angiograms. BfOP 1998; 1:29-32.

45 Hossack WJ, Boyce JF. Digital image processing introductory course. London: Dept Physics, King's College 1992:89-91.

46 Press WH, Flannery BP, Teukolsky SA, et al. Numerical recipes in C. Cambridge: Cambridge University Press 1991: 353-66. 